\section{A realidade virtual melhora o equilíbrio e o desempenho motor de uma criança com paralisia cerebral: relato de caso}

\section{Virtual reality improves balance and motor performance of a children with cerebral palsy}

\author{
Bianca Piriz Latorre, Maurício Tatsch Ximenes Carvalho, \\ Simone Rosa da Silva
}

\section{RESUMO}

\section{Autor correspondente:}

Nome: Bianca Piriz Latorre

E-mail: : bibi_latorre@hotmail.com

Telefone: (53) 99934- 1230

Formação Profissional: Bacharel

em Fisioterapia.

Filiação Institucional: Centro Universidade da Região da Campanha

Endereço para correspondência: Rua: Avenida Tupy Silveira

Bairro: Centro

Cidade: Bagé

Estado: Rio Grande do Sul

CEP: $964000-110$

Data de Submissão:

04/06/2019

Data de aceite:

16/09/2020

Conflito de Interesse: Não há conflito de interesse

\section{(cc) $\mathrm{BY}-\mathrm{NC}-\mathrm{ND}$}

O presente trabalho caracteriza-se como um relato de caso, cujo objetivo foi analisar os efeitos da A realização da coleta de dados foi conduzida a partir da seleção de uma criança com o diagnóstico de paralisia cerebral diparética espástica, atendida na Associação de Pais e Amigos dos Excepcionais (APAE) - Bagé- Rio Grande do Sul. As variáveis do estudo, aferidas previamente e após a intervenção, foram: o equilíbrio (Escala de Equilíbrio de BERG) e o desempenho motor (Gross Motor Function Measure - GMFM). A intervenção foi realizada através de atendimentos fisioterapêuticos baseados em realidade virtual através do Xbox®360 Kinect durante dois meses, duas vezes na semana no decorrer de uma hora. Os resultados sinalizaram um acréscimo no desempenho motor $(90,47 \%$ a $96,26 \%)$ e no equilíbrio (45 a 52 pontos) demonstrando os potenciais benefícios da terapia de realidade virtual.

PALAVRAS-CHAVE: Paralisia cerebral; Realidade virtual; Equilíbrio postural.

\section{ABSTRACT}

The present work is characterized as case report whose objective was to analyze the effects of virtual reality therapy therapy on the balance and motor performance of a child with Cerebral Palsy. Data collection was carried out from the selection of a children with spastic diparetic cerebral palsy, attended at the Association of Parents and Friends of the Exceptional (APAE) - Bagé-RS. The variables of the study, measured before and after the intervention, were: balance (Berg Balance Scale) and motor performance (Gross Motor Function Measure - GMFM). Intervention was performed through physiotherapeutic care based on virtual reality therapy by Xbox®360 Kinect for 2 months, 2 times a week in the course of 1 hour. Our findings showed an increase in motor performance $(90.47 \%$ to $96.26 \%$ ) and balance (45 to 52 points) demonstrating the potential benefits of virtual reality therapy.

KEYWORDS: Cerebral palsy; Virtual reality; Postural balance. terapia de realidade virtual no equilíbrio e no desempenho motor de uma criança com paralisia cerebral. 


\section{INTRODUÇÃO}

A paralisia cerebral (PC) é uma deficiência motora que ocorre na infância causada por uma lesão ou anomalia que afeta o cérebro imaturo ou em desenvolvimento, podendo ocorrer dentro do período pré-natal, perinatal ou pósnatal ${ }^{1}$. A incidência e a prevalência da PC se mantem constante em 1,5 a 2,5 para cada 1.000 nascidos vivos nos países desenvolvidos, e em países em desenvolvimento se mantem em sete para cada 1.000 nascidos vivos, fato que se deve, principalmente, às melhores condições de atendimento materno-infantil, diagnóstico e avanços tecnológicos².

A principal alteração observada nas crianças com PC é o comprometimento motor e de equilíbrio, com consequentes alterações na biomecânica corporal ${ }^{3}$. Os déficits motores da doença podem envolver partes distintas do corpo, resultando em classificações topográficas específicas (monoparesia ou monoplegia, diparesia ou diplegia, triparesia ou triplegia, hemiparesia ou hemiplegia e quadriplegia ou tetraparesia) ou alterações clínicas do tônus muscular (tipo espástico, discinético ou atetóide, atáxico, hipotônico e misto) ${ }^{4}$.

Os déficits motores da doença em relação a diparesia espástica, tem características predominantes de acometimento dos membros inferiores (MMII), principalmente da musculatura extensora e adutora, sendo os membros superiores pouco atingidos ${ }^{5}$. Portanto, alguns fatores que comprometem o desempenho motor das crianças com esse quadro são os déficits de equilíbrio estático e dinâmico, alinhamento corporal incorreto e controle postural anormal que comprometem atividades funcionais simples do cotidiano como: andar, correr, pular, dentre outras ${ }^{6}$.

A terapia baseada em realidade virtual $(\mathrm{RV})$ é uma ferramenta tecnológica capaz de treinar aspectos motores importantes como o equilíbrio, coordenação motora e conscientização corporal dentro de um contexto motivador. Esse tipo de reabilitação quando aplicada com continuidade e repetição, pode aperfeiçoar as habilidades motoras utilizando de diversos equipamentos comerciais, como videogames variados e o Xbox®360 Kinect ${ }^{7}$. A utilização deste e outros games proporciona aos participantes melhora nas capacidades motoras, agilidade, ajustes posturais, equilíbrio e força muscular de membros inferiores de forma lúdica e interativa, o que pode contribuir para a motivação, e ao mesmo tempo, conduzir à redução da apatia e absenteísmo em realizar 0 atendimento ${ }^{8}$.

Desta forma, o objetivo deste estudo foi avaliar o efeito da terapia de RV no equilíbrio e no desempenho motor de uma criança com PC Diparética Espástica, justificando a utilização dos jogos lúdicos que promovam terapias diferenciadas, e que proporcionem resultados em diversos aspectos da funcionalidade e aprendizagem motora.

\section{RELATO DE CASO}

O presente trabalho caracteriza-se como um relato de caso, cuja descrição segue as recomendações para descrição de relatos de casos - CARE (checklist). O estudo é de caráter quali-quantitativo, descritivo e foi aprovado pelo Comitê de Ética em Pesquisa local sob CAAE n 97355618.3.0000.5340. 


\section{Participante}

Selecionou-se uma criança com paralisia cerebral diparética espástica M.E.M.M do gênero feminino, de dez anos de idade, nível motor I segundo a GMFCS (Gross Motor Function Classification System) e padrão de rotação interna dos quadris, joelhos valgos e pés em equino, além de Transtorno de déficit de atenção e Hiperatividade (TDAH) associado à doença. Desde os cinco anos de idade recebe terapia de reabilitação clinica na Associação de Pais e Amigos dos Excepcionais - APAE/Bagé - RS, pela qual atingiu todos os marcos motores.

Mediante a assinatura do Termo de Consentimento Livre e Esclarecido (TCLE) aos Pais/Responsáveis a criança passou por uma anamnese e a mensuração de alguns testes referentes ao seu quadro motor antes do início da intervenção. Aplicou-se a escala de Ashoworth modificada em membros superiores (MMSS) e MMII mostrando espasticidade grau 1 predominante em MMIl e força muscular 4 na extremidade superior e inferior.

No exame físico apresenta mobilidade passiva e ativa preservadas em MMII e MMSS, reações de proteção e de equilíbrio testadas no sentido antêro-posterior, postêro-anterior e lateral com déficits no sentido lateral, equilíbrio dinâmico e estático apresentando dificuldades ao movimento, coordenação motora fina e grossa normais e a sensibilidade proprioceptiva, tátil e dolorosas preservadas.

Para a realização do estudo houve uma divisão da avaliação em dois períodos, um destinado a pré-intervenção e outro a pós-intervenção. Na pré-intervenção, realizou-se a classificação da criança conforme a GMFCS e a mensuração de duas escalas, referentes ao grau de equilíbrio (Escala de equilíbrio de Berg) e funcionalidade nas atividades motoras grossas - GMFM nas dimensões A, B, C, D e E.

\section{Desfechos}

\section{Avaliação do Desempenho motor}

A avaliação do desempenho motor foi realizada através do GMFM nas dimensões $A, B, C, D$ e $E$, durante aproximadamente 30 minutos. Os materiais utilizados para a aplicação do teste foram: cronômetro da marca vollo, fita métrica simples, cadeira com e sem braço, tatame, banco baixo e banco alto, brinquedos, escadas, bastão, bambolê e uma bola.

O protocolo GMFM, tem a finalidade de quantificar a funcionalidade motora grossa, através de um sistema de avaliação quantitativo contendo 88 itens com o propósito de mensurar alterações em crianças $\mathrm{PC}^{10}$. Esses itens são agrupados em cinco dimensões, sendo A: deitar e rolar, B: sentar, C: engatinhar e ajoelhar, D: ficar em pé e E: andar, correr e pular. Sua pontuação é obtida pela observação das crianças e graduada em uma escala de 4 pontos, na qual: 0 = não realiza, 1 = inicia atividade, 2 = completa parcialmente a atividade e $3=$ completa a atividade. 


\section{Avaliação do Equilíbrio}

A avaliação do equilíbrio se baseou na escala de equilíbrio de Berg, que foi aplicada no mesmo dia do GMFM, para não haver diferenças nos resultados. Os materiais utilizados para a aplicação do teste foram: cronômetro da marca vollo, fita métrica simples, cadeira com e sem braço, tatame, banco baixo e banco alto, brinquedos e uma bola.

A escala de equilíbrio de Berg se baseia em 14 itens comuns à vida diária, referentes ao grau de equilíbrio do indivíduo, a pontuação máxima é de 56 pontos, e cada item possui uma escala ordinal de cinco alternativas que variam de 0 a 4 pontos. Os pontos são baseados no tempo em que uma posição pode ser mantida, na distância em que 0 membro superior é capaz de alcançar à frente do corpo e no tempo para completar uma tarefa, entre outros ${ }^{11}$.

\section{Intervenção}

Após a realização da avaliação de pré-intervenção, foi dado início a terapia de realidade virtual utilizando-se do Xbox®360 Kinect, com o pacote de jogos Kinect Adventure. Os atendimentos foram realizados na sala de Estimulação precoce (GEP) na APAE/Bagé-RS e no domicilio da paciente, duas vezes por semana, com duração de 60 minutos e por um período de dois meses (agosto a outubro de 2018). A criança teve contato com dois jogos a cada sessão sendo eles a) Reflex Ridge, onde o jogador se mantem em cima de uma plataforma que se move sobre trilhos, devendo desviar de obstáculos pular e tocar esferas virtuais com o objetivo de movimentar os MMSS adequadamente para atingir as esferas, deslocamento do centro de gravidade do corpo lateralmente abaixando-se para esquivar-se dos obstáculos, além de saltar e do jogo b) River Rush, onde o jogador está sobre uma jangada em um rio, que deve ser controlada pelos movimentos do indivíduo com intuito de tocar as esferas virtuais no leito do rio, com o objetivo de deslocamentos láterolaterais do centro de gravidade do corpo, saltos e movimentos de MMSS, além de atenção a vários alvos e dupla tarefa. Durante o período de aplicação do protocolo a criança foi afastada das demais atividades que frequenta (fisioterapia convencional e equoterapia), com o objetivo de dar mais fidedignidade ao estudo.

A duração da sessão foi planejada para contemplar todo o tempo necessário para que fosse realizada anteriormente a terapia alongamentos passivos para reduzir a espasticidade de MMII (dos músculos de quadríceps, isquiotibiais, abdutores e adutores de quadril), além da montagem do aparelho do Xbox®360 Kinect que requeria alguns minutos adicionais para sua instalação e possíveis interrupções que pudessem atrapalhar o andamento do jogo. 


\section{RESULTADOS E DISCUSSÃO}

Efetuou-se um total de 22 sessões com a paciente (sendo a primeira e penúltima destinada à realização dos instrumentos de coleta de dados). Os resultados de pré e pós-intervenção referentes ao protocolo GMFM (Figura 1), coletados no início do tratamento e no penúltimo atendimento correspondente, foram os seguintes:

Figura 1 - Resultados do período pré e pós-intervenção referentes ao GMFM.

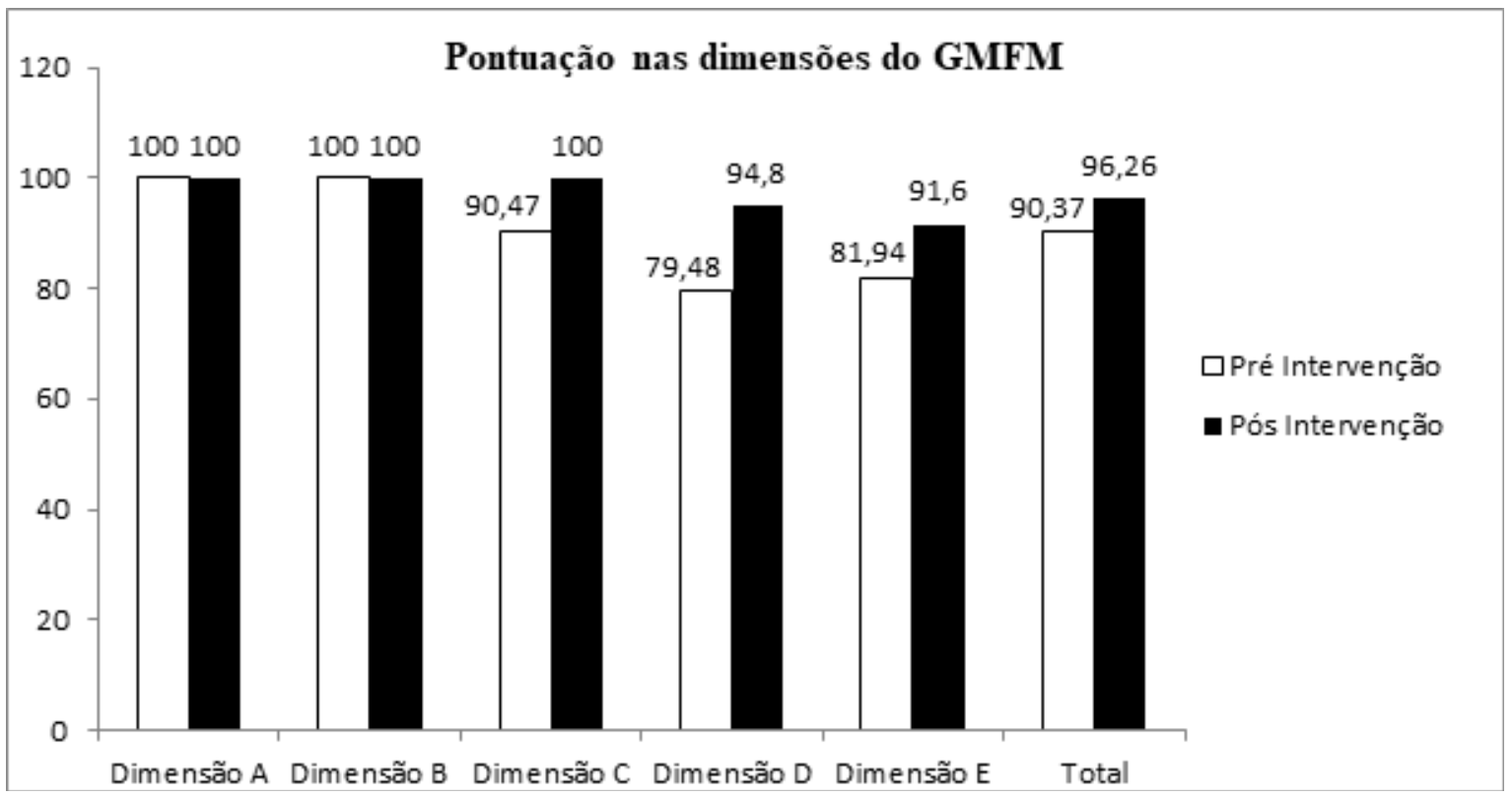

Na figura 1, percebe-se que na dimensão $A$ (deitar e rolar) e dimensão $B$ (sentar) a paciente manteve o escore de 100 pontos na pré e pós-intervenção. Contudo, nas dimensões C (engatinhar e ajoelhar), D (em pé) e E (andar, correr e pular) a paciente demostrou evolução nos escores da dimensão $C$ de 90,47 para 100 pontos, da dimensão $D$ de 79,48 para 94,6 pontos e na dimensão E de 81,94 para 91,6 pontos. Corroborando uma melhora considerável no escore total de $90,37 \%$ para $96,26 \%$ após a terapia.

Os achados estão em conformidade com estudos ${ }^{12,13,14}$ os quais relatam acerca da eficácia da RV no desempenho de atividades motoras em crianças com PC diparética espástica, demonstrando melhora geral na funcionalidade e na coordenação motora avaliada pelo GMFM de $83,73 \%$ para 86,59 , além da melhora do equilíbrio, avaliado pela escala Pediatric Balance Scale (adaptação da escala de Berg para crianças), melhora na percepção visual, controle postural, alinhamento do centro de gravidade e distribuição de peso médio-lateral mais simétrico após a reabilitação.

Os resultados referentes ao equilíbrio, avaliado através da escala de equilíbrio de Berg (escore total) são apresentados na figura 2. 
Figura 2 - Resultados do período pré e pós-intervenção da Escala de Equilíbrio de Berg.



Desta forma, o escore total de pré intervenção foi de 45 pontos descritos na escala de equilíbrio de Berg como indicativos de déficits de equilíbrio e alto risco para quedas, para 52 pontos após a terapia. Evidenciando os benefícios da intervenção com o protocolo de RV no equilíbrio para realização de inúmeras atividades de vida diária do cotidiano da criança.

Estudos relatam ${ }^{14,15}$ a melhora no equilíbrio de pacientes com paralisia cerebral avaliados pela escala de Berg utilizando a RV, de 35 pontos para 46 pontos, demonstrando melhora no equilíbrio estático e dinâmico. Salienta-se que outros estudos de intervenção em pacientes com Acidente Vascular Encefálico e Doença de Parkinson, apresentaram resultados satisfatórios após o uso de RV tanto isolada como associada a terapia fisioterapêutica, com a melhora do equilíbrio, da força muscular e da cinemática da marcha, confirmando, assim, os resultados apresentados ${ }^{10,14}$.

É importante enfatizar os benefícios observados com a escolha dos jogos dentro da intervenção com RV e do console do Xbox®360 Kinect. Um estudo realizou um trabalho com 28 crianças (diparéticas e hemiparéticas espásticas) com idades entre 3 e 12 anos encaminhadas pelo Serviço de Fisiatria e Reabilitação do Hospital de Clínicas de Porto Alegre e avaliadas como estando nos níveis I, II e III pelo Sistema de Classificação da Função Motora Grossa. Os participantes foram submetidos a 16 sessões de 40 minutos em que eram executados dois jogos do pacote Kinect Adventure dentro do Xbox®360 Kinect em um período de 8 semanas. Os resultados demonstraram melhora da função motora grossa com aumento significativo nos escores finais avaliados pela escala de Medida da Função Motora Grossa 
$(p<0,05)$ aplicada antes e após a intervenção. Estes achados sugerem viabilidade para uso dos jogos interativos do sistema Xbox360 Kinect@ na recuperação motora de crianças com PC, conforme o presente trabalho ${ }^{13}$.

Como limitações cita-se, primeiro, algumas modificações realizadas durante o protocolo de atendimento, pois optou-se pela realização dos jogos com o uso do acessório do tipo sling para evitar o aumento do padrão de rotação interna dos quadris e segundo observou-se que a paciente pelo quadro de TDAH associado a PC tinha uma dispersão em relação ao ambiente, interrompendo os jogos em alguns momentos para outras atividades.

\section{CONSIDERAÇÕES FINAIS}

Os achados deste estudo demonstraram os benefícios da terapia de realidade virtual através do Xbox®360 Kinect no equilíbrio e no desempenho motor de uma criança com PC diparética espástica. Dessa forma, a aplicabilidade clínica dos resultados do presente estudo reside no fato de que a RV contribui no tratamento da PC de forma inovadora através da utilização dos jogos lúdicos na função motora e no equilíbrio. A significância clínica desses achados deve ser respaldada através de ensaios clínicos randomizados.

\section{REFERÊNCIAS}

1. Gulati S, Sondhi V. Cerebral palsy: an overview. Indian J. Pediatr. 2018; 85(11):1006-1016.

2. Xavier MJ, Rodrigues NMNM, Araujo MB. Realidade virtual na reabilitação da paralisia cerebral: Um estudo de caso. Braz. J. of Develop. 2018; 6(7):47002-47011.

3. Vasconcelos RLM, et al. Avaliação do desempenho funcional de crianças com paralisia cerebral de acordo com níveis de comprometimento motor. Rev. Bras. Fisioter. 2009; 13(5):390.

4. Duarte PHM, et al. A realidade virtual como instrumento de apoio à conduta fisioterapêutica.

Arch Health Invest. 2018; 7(2):59-61.

5. Bondan DE. Realidade virtual na fisioterapia: utilização para crianças com paralisia cerebral: revisão da literatura. Revista Contexto \& Saúde. 2016; 16(31), 79-88. 
6. Brito VB, et al. Os benefícios que a realidade virtual oferece no tratamento de indivíduos com paralisia cerebral. Jornada de Fisioterapia do Sertão Central. 2018; 1(1).

7. Arnoni JLB, et al. Efeito da intervenção com videogame ativo sobre o autoconceito, equilíbrio, desempenho motor e sucesso adaptativo de crianças com paralisia cerebral: estudo preliminar. Fisioter. Pesqui. 2018; 25(3):294-302.

8. Jesus ES, et al. Gameterapia na reabilitação de pacientes com paralisia cerebral. Revista Brasileira de Saúde Funcional. 2018; 1(1):9.

9. Silva DBR, Dias LB, Pfeifer LI. Confiabilidade do Sistema de Classificação da Função Motora Grossa Ampliado e Revisto (GMFCS E \& R) entre estudantes e profissionais de saúde no Brasil. Fisioter. Pesqui. 2016; 23(2):142-147.

10. Santos CT. Eficácia da realidade virtual e dos principais games utilizados no tratamento da paralisia cerebral. Revista de Saúde ReAGES. 2019; 2(4):6-10.

11. Caiana TL, Nogueira DL, Lima ACDA. A realidade virtual e seu uso como recurso terapêutico ocupacional: revisão integrativa. Cad. Ter. Ocup. 2016; 24(3): 575-589.

12. Oliveira NM, Fornazza GB, De Sousa TQ. A realidade virtual como recurso terapêutico para crianças com paralisia cerebral: uma revisão de literatura. Revista Pesquisa e Ação. 2018; 4(3).

13. Santos Júnior FFU, et al. Efeitos de uma intervenção com realidade virtual no controle motor de uma criança com paralisia cerebral: um relato de caso. Motricidade. 2018; 14(1):351-354.

14. De Souza VPS, et al. repercussões da realidade virtual sobre o equilíbrio, tônus muscular e qualidade de vida de crianças com encefalopatia crônica não evolutiva da infância. Revista CPAQV-Centro de Pesquisas Avançadas em Qualidade de Vida-CPAQV Journal. 2020; 12(1).

15. Kintschner, NR. Efeitos de um programa de gameterapia controlada por leap motion na função manual de adultos com paralisia cerebral. 2020. 64 f. Dissertação (Mestrado em Distúrbios do Desenvolvimento) - Universidade Presbiteriana Mackenzie, São Paulo, 2020. 